\title{
Patterns of cutaneous nerve fibre loss and regeneration in type 2 diabetes with painful and painless polyneuropathy
}

\author{
Gidon J. Bönhof $^{1} \cdot$ Alexander Strom $^{1,2} \cdot$ Sonja Püttgen $^{1} \cdot$ Bernd Ringel $^{1} \cdot$ \\ Jutta Brüggemann ${ }^{1}$ • Kálmán Bódis ${ }^{1}$ • Karsten Müssig ${ }^{1,2,3}$ • Julia Szendroedi ${ }^{1,2,3}$. \\ Michael Roden ${ }^{1,2,3} \cdot$ Dan Ziegler ${ }^{1,2,3}$
}

Received: 13 June 2017 / Accepted: 2 August 2017 / Published online: 15 September 2017

(C) Springer-Verlag GmbH Germany 2017

\begin{abstract}
Aims/hypothesis The determinants and mechanisms of the development of diabetic sensorimotor polyneuropathy as a painful (DSPN+p) or painless (DSPN-p) entity remain unclear. We examined the degree of cutaneous nerve fibre loss and regeneration in individuals with type 2 diabetes with DSPN+p or DSPN-p compared with individuals with recent-onset type 2 diabetes and corresponding healthy volunteers.

Methods In this cross-sectional study, skin biopsies taken from the distal lateral calf were obtained from individuals with recent-onset type 2 diabetes $(n=32)$ from the German Diabetes Study, with DSPN+p $(n=34)$ and DSPN-p $(n=32)$ from the PROPANE study, and volunteers with normal glucose tolerance $(n=50)$. Double immunofluorescence staining for protein gene product 9.5 (PGP9.5) (pan-neuronal marker) and growth-associated protein 43 (GAP-43) (nerve regeneration marker) was applied to assess intraepidermal nerve fibre density (IENFD) and length (IENFL) and dermal nerve fibre length (DNFL). DSPN was diagnosed using the modified Toronto Consensus (2011) criteria, while neuropathic pain was assessed using an 11-point Numerical Rating Scale.

Results After adjustment for age, sex, $\mathrm{BMI}$ and $\mathrm{HbA}_{1 \mathrm{c}}$, IENFD and IENFL were reduced for both markers in
\end{abstract}

Dan Ziegler

dan.ziegler@ddz.uni-duesseldorf.de

1 Institute for Clinical Diabetology, German Diabetes Center (DDZ), Leibniz Center for Diabetes Research at Heinrich Heine University, Auf'm Hennekamp 65, 40225 Düsseldorf, Germany

2 German Center for Diabetes Research (DZD), Munich, Neuherberg, Germany

3 Division of Endocrinology and Diabetology, Medical Faculty, Heinrich Heine University, Düsseldorf, Germany individuals with recent-onset diabetes and both DSPN groups compared with control participants (all $p<0.05$ ), but did not differ between the DSPN groups. The DNFL GAP-43/PGP9.5 ratio was higher in the DSPN+p and DSPN-p groups compared with control participants $(1.18 \pm 0.28$ and $1.07 \pm 0.10 \mathrm{vs}$ $1.02 \pm 0.10 ; p \leq 0.05)$ and in the DSPN + p group compared with DSPN-p $(p<0.05)$. Correlation analyses showed distinct inverse associations between the DNFL GAP-43/PGP9.5 ratio and PGP9.5 positive IENFD as well as DNFL (IENFD: $\beta=-0.569$, DNFL: $\beta=-0.639$; both $p<0.0001$ ) in individuals with type 2 diabetes, but not in the control group. A similar pattern was found for correlations between the DNFL GAP-43/PGP9.5 ratio and peripheral nerve function tests.

Conclusions/interpretation Dermal nerve fibre regeneration is enhanced in DSPN, particularly in DSPN $+p$, and increases with advancing intraepidermal nerve fibre loss. These data suggest that, despite progressive epidermal fibre loss, dermal nerve repair is preserved, particularly in DSPN+p, but fails to adequately counteract epidermal neurodegenerative processes.

Keywords Nerve regeneration - Neuropathic pain . Neuropathy $\cdot$ Skin biopsy $\cdot$ Type 2 diabetes

$\begin{array}{ll}\text { Abbreviations } \\ \text { DNFL } & \text { Dermal nerve fibre length } \\ \text { DSPN } & \text { Diabetic sensorimotor polyneuropathy } \\ \text { DSPN+p } & \text { Painful diabetic sensorimotor polyneuropathy } \\ \text { DSPN-p } & \text { Painless diabetic sensorimotor polyneuropathy } \\ \text { GAP-43 } & \text { Growth-associated protein-43 } \\ \text { GDS } & \text { German Diabetes Study } \\ \text { IENFD } & \text { Intraepidermal nerve fibre density } \\ \text { IENFL } & \text { Intraepidermal nerve fibre length }\end{array}$




$\begin{array}{ll}\text { NCV } & \text { Nerve conduction velocity } \\ \text { NDS } & \text { Neuropathy Disability Score } \\ \text { NRS } & \text { Numerical Rating Scale } \\ \text { NSS } & \text { Neuropathy Symptom Score } \\ \text { PGP9.5 } & \text { Protein gene product } 9.5 \\ \text { PROPANE } & \text { Probing the Role of Sodium Channels in } \\ & \text { Painful Neuropathies } \\ \text { SFN } & \text { Small fibre neuropathy } \\ \text { SNAP } & \text { Sensory nerve action potential } \\ \text { TDT } & \text { Thermal detection threshold } \\ \text { VPT } & \text { Vibration perception threshold }\end{array}$

\section{Introduction}

Diabetic sensorimotor polyneuropathy (DSPN) affects approximately $30 \%$ of people with diabetes and its sequelae, such as neuropathic pain and foot ulcers, contribute to substantial morbidity, high socioeconomic burden, reduced quality of life and increased mortality rates $[1,2]$. Since the mechanisms contributing to the development of DSPN as a painful $(\mathrm{DSPN}+\mathrm{p})$ and painless (DSPN-p) entity are not well understood, the current options to prevent and treat either are limited $[3,4]$.

In diabetic neuropathies, axonal atrophy, demyelination, loss of nerve fibres and blunted regeneration of nerve fibres are present, and both nerve regeneration as well as degeneration contribute to nerve pathology [4]. Nerve damage is followed by a series of morphological and molecular changes in the perikarya of injured nerves into a regenerative state, priming neurons for regrowth [5]. Therefore, further understanding of neural plasticity, regenerative capacity and nerve fibre degeneration is required to identify novel therapeutic and preventive approaches specific for the two types of DSPN.

Although small nerve fibre damage is considered a prerequisite for the presence of neuropathic pain in diabetes, there is no definite evidence of an exclusive or prominent involvement of small fibres in DSPN+p, and biomarkers to predict the development of neuropathic pain are still lacking [6]. Similarities and differences between DSPN+p and DSPN-p have been described, but the conundrum of why some individuals with DSPN develop neuropathic pain while others do not has not been solved $[6,7]$.

Skin biopsy is a reliable, minimally invasive tool for the assessment of epidermal and dermal nerve fibres in peripheral nerve disorders [8]. The pan-neuronal marker protein gene product 9.5 (PGP9.5), a member of the ubiquitin hydroxylase system, is widely used to detect and quantify cutaneous nerve fibres [9]. Using this technique, studies have shown that small-calibre nerve fibres penetrating the epidermis, which are mostly nociceptive [8], are already reduced in the early stages of type 2 diabetes [10] and are diminished in small fibre neuropathies (SFNs) of different origins, including diabetes $[8,11]$.

Growth-associated protein 43 (GAP-43) is a membrane protein that is involved in the process of nerve regeneration $[12,13]$. As it is a major constituent in axonal growth cones after nerve injury [4], GAP-43 is expressed in peripheral nerve fibre areas with high neural plasticity such as the epidermis and dermis. Hence, it can be used as a marker for regenerating nerve fibres $[14,15]$.

Several studies have reported that nerve regeneration may be altered in diabetes mellitus with and without DSPN, but the varying results due to small population sizes, heterogeneous study populations and methodological differences do not give a conclusive answer about regenerative capacity and the variable by which it is reflected best [16-19]. Here we determined the degree of cutaneous nerve fibre loss and regeneration in individuals with type 2 diabetes with DSPN+p and DSPN-p, compared with individuals with recent-onset diabetes and corresponding control participants. We hypothesised that the patterns of cutaneous nerve fibre loss and repair may differ between the two types of DSPN, as well as between individuals with recent-onset diabetes and normal glucose tolerance.

\section{Methods}

Participant selection This cross-sectional study was conducted in accordance with the Declaration of Helsinki and was approved by the ethics committee of Heinrich Heine University, Düsseldorf, Germany. After obtaining written informed consent, 98 participants with type 2 diabetes, defined according to the ADA criteria [20], were allocated to three groups: recent-onset type 2 diabetes $(n=32)$ DPSN-p $(n=32)$, and DSPN $+\mathrm{p}(n=34)$. The presence of pain in the distal lower extremities lasting $\geq 1$ year with a pain intensity $\geq 4$ (24 $\mathrm{h}$ average or maximum) on the 11-point Numerical Rating Scale (NRS) in the absence of analgesic treatment, or according to the medical history (recall and/or records) prior to analgesic treatment, was used to define DSPN+p. Individuals with DSPN-p reported a pain intensity on the $24 \mathrm{~h}$ average NRS of 0 , except for two who had an NRS of 1 without analgesic treatment. Pain not associated with DSPN due to conditions such as low back pain or osteoarthritis was not considered in the pain assessment $[21,22]$. There was a difference in age between the recent-onset group and the DSPN groups; therefore, two glucose-tolerant control groups of similar age and sex were formed from 50 individuals with normal glucose tolerance. The Control 1 group which included all 50 individuals was used for comparison with the recent-onset group. The Control 2 group included the 25 oldest individuals from the Control 1 group for comparison with the DSPN groups. Individuals with recent-onset type 2 diabetes and 
control participants were recruited from the German Diabetes Study (GDS), which is a prospective longitudinal cohort study investigating the impact of subphenotypes on the course of the disease (ClinicalTrials.gov Identifier: NCT01055093). The study design and cohort profile of the GDS have been published elsewhere [23]. Individuals with type 2 diabetes with DSPN were recruited from the Probing the Role of Sodium Channels in Painful Neuropathies (PROPANE) study, an observational study investigating the factors that contribute to the development of neuropathic pain (ClinicalTrials.gov Identifier: NCT02243475) [24]. Inclusion criteria for entry into the PROPANE study for the present cohort were age $\geq 18$ years, type 1 or type 2 diabetes [20], and a diagnosis of sensory, sensorimotor and/or small fibre DSPN as possible, probable or confirmed according to the Toronto Consensus criteria [21]. Exclusion criteria were other causes of neuropathy (hypothyroidism, renal failure, vitamin $\mathrm{B}_{12}$ deficiency, monoclonal gammopathy, alcohol abuse [ $>5 \mathrm{U} /$ day], malignancies, drugs known to cause neuropathy) and concomitant diseases that might interfere with the participant's ability to fill in questionnaires. No biopsies were taken from individuals on anticoagulant therapy or with peripheral artery disease, chronic infections of the lower extremities or presenting other contraindications. All assessments in individuals from the PROPANE study were performed specifically for the present study, while data from some individuals from the GDS related to neurological examination and peripheral nerve function had been published previously [10].

Peripheral nerve function Electrophysiological testing, quantitative sensory testing and neuropathy score surveys were performed as previously described [10]. Motor nerve conduction velocity (NCV) was measured in the peroneal nerve, while sensory NCV and sensory nerve action potentials (SNAPs) were determined in the sural nerve at a skin temperature of 33-34 ${ }^{\circ} \mathrm{C}$ using surface electrodes (Nicolet VikingQuest, Natus Medical, San Carlos, CA, USA). Vibration perception threshold (VPT) was measured at the medial malleolus using the method of limits (Vibrameter, Somedic, Stockholm, Sweden). Thermal detection thresholds (TDTs) to warm and cold stimuli were determined on the dorsum of the foot using the method of limits (TSA-II NeuroSensory Analyzer, Medoc, Ramat Yishai, Israel). Neurological examination was performed using the Neuropathy Disability Score (NDS) [25]. Neuropathic symptoms were assessed by the Neuropathy Symptom Score (NSS) [25] and the NRS for neuropathic pain average over $24 \mathrm{~h}$ and maximum scores.

Skin biopsy Skin punch biopsy specimens $(3 \mathrm{~mm})$ were taken under local anaesthesia (Meaverin-Actavis 1\%, 1-1.8 ml, Actavis, Langenfeld, Germany) from the left distal lateral calf, about $10 \mathrm{~cm}$ proximal to the lateral malleolus. Details of sampling and processing of the biopsy have been published previously $[26,27]$. In brief, after fixation with $2 \%$ periodatelysine-paraformaldehyde at $4{ }^{\circ} \mathrm{C}$ for $24 \mathrm{~h}$ tissue was rinsed twice for $10 \mathrm{~min}$ with $0.1 \mathrm{~mol} / \mathrm{l}$ Sorensen buffer. Following incubation in 33\% sucrose for $3 \mathrm{~h}$ the specimens were incubated with $0.02 \mathrm{~mol} / 1$ Sorensen buffer containing $20 \%$ glycerol at $4^{\circ} \mathrm{C}$ overnight, embedded and stored at $-80^{\circ} \mathrm{C}$.

Immunofluorescence staining The double immunofluorescence staining for PGP9.5 and GAP-43 was performed using three $50 \mu \mathrm{m}$ sections for each individual using the freefloating method. After blocking with 5\% BSA and 5\% normal goat serum for $30 \mathrm{~min}$, sections were incubated with the rabbit polyclonal anti-PGP9.5 (AB1761, 1:3000, Merck Millipore, Billerica, MA, USA) and the mouse anti-GAP-43 (MAB347, 1:1000, Merck Millipore) antibodies overnight. Thereafter, sections were incubated with Alexa488 conjugated goat antirabbit IgG (A-11034, 1:500, Thermo Fisher Scientific, Waltham, MA, USA) and Alexa555 conjugated goat antimouse IgG (A-21424, 1:500, Thermo Fisher Scientific) secondary antibodies for $1 \mathrm{~h}$. Nuclei were stained with Hoechst $33,342(1 \mathrm{mg} / \mathrm{ml})$ for $5 \mathrm{~min}$. Image stacks were acquired using a Nikon Ti-E inverted microscope (Nikon, Minato, Japan) equipped with a DS-Qi2 digital monochrome camera, corresponding filters and a x20/0.75 CFI P-Apo DM objective. Each antibody was validated separately prior to use in double immunofluorescence.

Image analysis After focus stacking and general brightness and contrast adjustment, images were analysed using the NISElements AR v4.50.00 (Nikon) or ImageJ 1.49 (National Institutes of Health, Bethesda, MD, USA) software. For each individual and marker, three sections were analysed. Intraepidermal nerve fibre density (IENFD) was determined as described previously [18]. To determine intraepidermal nerve fibre length (IENFL) and dermal nerve fibre length (DNFL), the epidermal and dermal areas of interest were defined. The epidermal area of interest was defined using nucleus staining. The dermal area of interest was limited by the epidermal-dermal junction and a line $200 \mu \mathrm{m}$ below the dermal-epidermal junction using the $25 \%$ method [28]. Then, the lengths of the fibres within these areas were measured and the total fibre length $(\mu \mathrm{m})$ was divided by the total corresponding area $\left(\mathrm{mm}^{2}\right)$. Thicker nerve fibre bundles were considered as fibres and measured likewise. IENFD, IENFL and DNFL for each individual were expressed as the means of three analysed sections. GAP-43/PGP9.5 ratios for each of these three measures were obtained by calculating the proportion of GAP-43 to PGP9.5 positive fibres. Representative images of a doubleimmunofluorescent stained section with indication of intraepidermal and dermal nerve fibres in an individual with normal glucose tolerance are shown in Fig. 1. Microscopy and image analysis were performed with anonymised samples and the examiners were blinded to group allocation. 

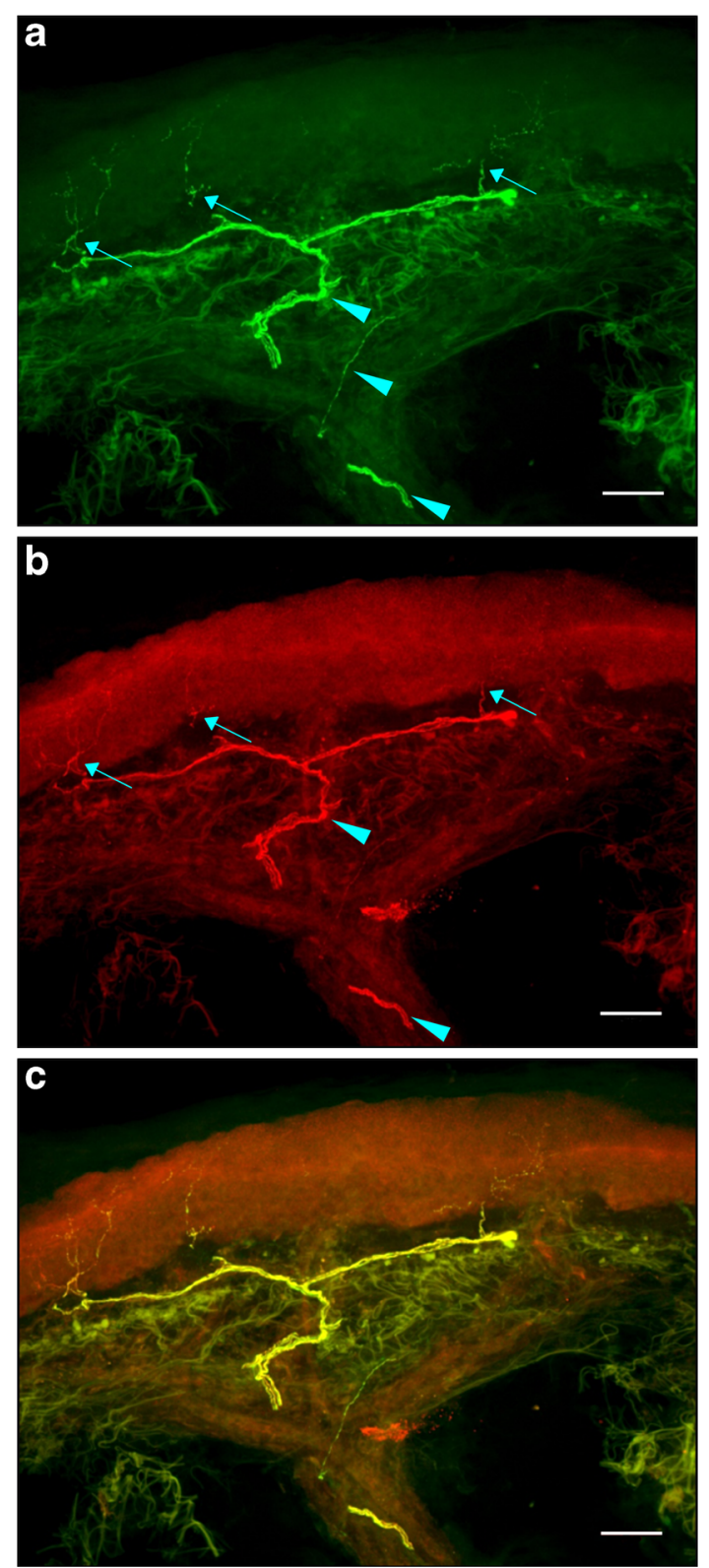

Fig. 1 Double immunofluorescence with PGP9.5 and GAP-43. Detection of (a) PGP9.5 (green), (b) GAP-43 (red) and (c) positive nerve fibres in skin biopsies (yellow; PGP9.5 and GAP-43 merged). Arrows, intraepidermal nerve fibre; arrow heads, dermal nerve fibre. Scale bars, $50 \mu \mathrm{m}$

Statistical analysis Categorical data are expressed as percentages of participants. Continuous data are expressed as mean \pm SD. Categorical variables were compared using the $\chi^{2}$ test. For normally distributed data, parametric tests were used (Student's $t$ test or Pearson correlation), otherwise nonparametric tests (Mann-Whitney $U$ test or Spearman rank correlation) were applied. All group comparisons were adjusted for sex, age and BMI, except for the skin biopsy measures which were additionally adjusted for $\mathrm{HbA}_{1 \mathrm{c}}$. To determine possible associations between two variables, multiple linear regression analyses with adjustments for sex, age and BMI were performed. Variables that were not normally distributed were $\log _{\mathrm{e}}$-transformed before being entered into the regression models. The level of significance was set at $\alpha=0.05$. All analyses were performed with SPSS Statistics for Windows, Version 22.0 (IBM, Armonk, NY, USA). All graphs were generated using GraphPad Prism, Version 6.04 (GraphPad Software, La Jolla, CA, USA).

\section{Results}

The demographic, neurophysiological and clinical characteristics of the groups studied are shown in Table 1. All three diabetes groups showed a higher BMI compared with the corresponding control participants. The DSPN-p group were older compared with the Control 2 group, while those with DSPN + p were younger than those with DSPN-p. Both DSPN groups had higher total cholesterol and $\mathrm{HbA}_{1 \mathrm{c}}$ levels compared with the Control 2 group (all $p<0.05$ ). Among the individuals with recent-onset type 2 diabetes, $21.9 \%$ had DSPN-p, while none had DSPN+p. In the DSPN+p/DSPN-p groups, $47.1 \% / 6.3 \%$ were using alpha2-delta ligands (gabapentin or pregabalin), $8.8 \% / 0 \%$ were using serotonin noradrenaline (norepinephrine) reuptake inhibitors (duloxetine or venlafaxine), $8.8 \% / 3.1 \%$ were using opiates and $8.8 \% / 0 \%$ were using other analgesics for neuropathic pain.

After adjustment for age, sex and BMI, peroneal motor, sural sensory NCV were reduced in both DSPN groups compared with the Control 2 group. Sural SNAPs were reduced in the recent-onset and DSPN groups compared with the corresponding control participants. The VPTs were reduced in the DSPN groups compared with the Control 2 group. Warm TDTs were reduced in the DSPN+p and cold TDTs were reduced in both DSPN compared with Control 2 groups. NSS and NDS were higher in the DSPN groups compared with the Control 2 group. As expected, NSS was higher in the DSPN+p than in the DSPN-p groups, and the average and maximum pain scores were higher in individuals with DSPN $+\mathrm{p}$ compared with DSPN-p and the Control 2 group (all $p<0.05$ ).

Table 2 shows the cutaneous nerve fibre density and length in the five groups studied. After adjustment for age, sex, BMI and $\mathrm{HbA}_{1 \mathrm{c}}$, both DSPN groups showed reduced IENFD and IENFL compared with the Control 2 group for both markers $(p<0.05)$, while no differences were found between the DSPN $+p$ and DSPN-p groups. IENFD and IENFL were also reduced in the recent-onset group compared with the Control 1 group $(p<0.05)$. Differences in DNFL were not found between the recent-onset and Control 1 groups or between the DSPN-p and the Control 2 groups or between the DSPN groups. No between-group differences in the GAP-43/PGP9.5 ratio for IENFD or IENFL were noted. The DNFL GAP-43/ 
Table 1 Demographic, neurophysiological and clinical characteristics of the study participants

\begin{tabular}{|c|c|c|c|c|c|}
\hline & Control 1 & $\begin{array}{l}\text { Recent-onset } \\
\text { type } 2 \text { diabetes }\end{array}$ & Control 2 & DSPN-p & $\mathrm{DSPN}+\mathrm{p}$ \\
\hline$n$ & 50 & 32 & 25 & 32 & 34 \\
\hline Sex (\% male) & 84.0 & 75.0 & 92.0 & 90.6 & 76.5 \\
\hline Age (years) & $53.0 \pm 16.0$ & $51.0 \pm 10.1$ & $64.9 \pm 4.9$ & $71.3 \pm 8.1^{\dagger}$ & $67 \pm 8.6^{\ddagger}$ \\
\hline Height (cm) & $178 \pm 8$ & $177 \pm 7$ & $179 \pm 9$ & $177 \pm 8$ & $175 \pm 7$ \\
\hline BMI $\left(\mathrm{kg} / \mathrm{m}^{2}\right)$ & $27.4 \pm 5.8$ & $32.5 \pm 5.5^{*}$ & $26.3 \pm 3.0$ & $28.9 \pm 4.1^{\dagger}$ & $31.0 \pm 4.4^{\dagger}$ \\
\hline $\begin{array}{l}\text { Diabetes duration } \\
\text { (years) }\end{array}$ & - & $0.2 \pm 0.4$ & - & $15.2 \pm 10.7$ & $15.7 \pm 9.9$ \\
\hline Current smoker (\%) & 16.0 & 16.1 & 12.0 & 6.3 & 20.6 \\
\hline Heart rate (bpm) & $66 \pm 9$ & $72 \pm 11$ & $67 \pm 10$ & $70 \pm 11$ & $71 \pm 10$ \\
\hline Systolic BP (mmHg) & $127 \pm 17$ & $132 \pm 15$ & $134 \pm 17$ & $136 \pm 18$ & $138 \pm 16$ \\
\hline Diastolic BP (mmHg) & $72 \pm 10$ & $74 \pm 11$ & $76 \pm 8$ & $69 \pm 9$ & $72 \pm 9$ \\
\hline $\begin{array}{l}\text { Total cholesterol } \\
\qquad(\mathrm{mmol} / \mathrm{l})\end{array}$ & $5.25 \pm 0.85$ & $5.43 \pm 1.06$ & $5.95 \pm 0.44$ & $4.91 \pm 1.27$ & $4.94 \pm 1.01$ \\
\hline $\begin{array}{l}\text { HDL-cholesterol } \\
\qquad(\mathrm{mmol} / \mathrm{l})\end{array}$ & $1.50 \pm 0.51$ & $1.31 \pm 0.36$ & $1.41 \pm 0.28$ & $1.35 \pm 0.38$ & $1.36 \pm 0.39$ \\
\hline $\begin{array}{l}\text { LDL-cholesterol } \\
(\mathrm{mmol} / \mathrm{l})\end{array}$ & $3.34 \pm 0.85$ & $3.52 \pm 0.93$ & $3.90 \pm 0.41$ & $3.00 \pm 1.14$ & $3.08 \pm 0.88$ \\
\hline $\begin{array}{l}\text { C-reactive protein } \\
(\mathrm{nmol} / \mathrm{l})^{\mathrm{a}}\end{array}$ & $14.3 \pm 13.3$ & $39.0 \pm 28.6^{*}$ & - & $22.4 \pm 24.5$ & $32.4 \pm 30.5$ \\
\hline $\mathrm{HbA}_{1 \mathrm{c}}(\%)$ & $5.24 \pm 0.30$ & $6.42 \pm 1.00 *$ & $5.36 \pm 0.24$ & $7.31 \pm 0.88^{\dagger}$ & $7.40 \pm 1.32^{\dagger}$ \\
\hline $\mathrm{HbA}_{1 \mathrm{c}}(\mathrm{mmol} / \mathrm{mol})$ & $33.78 \pm 3.29$ & $46.65 \pm 10.96^{*}$ & $35.05 \pm 2.67$ & $56.39 \pm 9.57^{\dagger}$ & $57.34 \pm 14.40^{\prime}$ \\
\hline $\begin{array}{l}\text { Peroneal motor NCV } \\
(\mathrm{m} / \mathrm{s})^{\mathrm{a}}\end{array}$ & $45.4 \pm 4.5$ & $44.0 \pm 4.8$ & $44.3 \pm 3.4$ & $38.2 \pm 6.42^{\dagger}$ & $38.3 \pm 6.42^{\dagger}$ \\
\hline $\begin{array}{l}\text { Sural sensory NCV } \\
(\mathrm{m} / \mathrm{s})^{\mathrm{a}}\end{array}$ & $45.0 \pm 4.6$ & $44.9 \pm 5.8$ & $44.2 \pm 4.0$ & $33.6 \pm 7.22^{\dagger}$ & $32.5 \pm 8.12^{\dagger}$ \\
\hline Sural SNAP $(\mu \mathrm{V})^{\mathrm{a}}$ & $11.0 \pm 5.8$ & $7.2 \pm 3.81$ & $10.3 \pm 6.3$ & $4.6 \pm 3.82^{\dagger}$ & $3.1 \pm 3.42^{\dagger}$ \\
\hline Malleolar VPT $(\mu \mathrm{m})^{\mathrm{a}}$ & $1.7 \pm 1.8$ & $2.11 \pm 2.74$ & $2.1 \pm 2.1$ & $11.2 \pm 8.32^{\dagger}$ & $10.1 \pm 8.42^{\dagger}$ \\
\hline $\begin{array}{l}\text { Foot warm TDT } \\
\left({ }^{\circ} \mathrm{C}\right)^{\mathrm{a}}\end{array}$ & $39.1 \pm 3.8$ & $40.5 \pm 4.0$ & $40.0 \pm 3.9$ & $44.3 \pm 5.5$ & $45.5 \pm 5.32^{\dagger}$ \\
\hline Foot cold TDT $\left({ }^{\circ} \mathrm{C}\right)^{\mathrm{a}}$ & $27.8 \pm 4.1$ & $26.3 \pm 6.6$ & $27.1 \pm 3.7$ & $19.0 \pm 10.32^{\dagger}$ & $15.9 \pm 12.52^{\dagger}$ \\
\hline $\mathrm{NSS}^{\mathrm{a}}$ & $0.12 \pm 0.85$ & $0.78 \pm 2.18$ & $0.24 \pm 1.20$ & $5.84 \pm 2.08^{\dagger}$ & $7.11 \pm 1.41^{\dagger,+}$ \\
\hline $\mathrm{NDS}^{\mathrm{a}}$ & $0.78 \pm 1.05$ & $1.41 \pm 1.64$ & $1.27 \pm 1.10$ & $5.65 \pm 2.88^{\dagger}$ & $6.83 \pm 2.64^{\dagger}$ \\
\hline $\begin{array}{l}\text { Average NRS pain } \\
\text { score (points) }\end{array}$ & 0 & 0 & 0 & $0.06 \pm 0.25$ & $\begin{array}{l}3.32 \pm 2.61^{\dagger, \star} \\
\left(4.34 \pm 3.02^{\mathrm{b}}\right)\end{array}$ \\
\hline $\begin{array}{l}\text { Maximum NRS pain } \\
\text { score (points) }{ }^{\mathrm{a}}\end{array}$ & 0 & 0 & 0 & $0.27 \pm 0.89$ & $\begin{array}{l}5.43 \pm 2.67^{\dagger,}+ \\
\left(6.44 \pm 2.80^{\mathrm{b}}\right)\end{array}$ \\
\hline
\end{tabular}

${ }^{*} p<0.05$ vs Control $1,{ }^{\dagger} p<0.05$ vs Control $2,{ }^{\dagger} p<0.05$ vs DSPN-p

${ }^{\mathrm{a}}$ Adjusted for sex, age and BMI

${ }^{\mathrm{b}}$ From medical history prior to analgesic treatment
PGP9.5 ratio was higher in both DSPN groups compared with the Control 2 group $(p<0.05)$. Moreover, the DNFL GAP-43/ PGP9.5 ratio was higher in the DSPN+p compared with the DSPN-p group $(p<0.05)$. The number of participants showing a DNFL GAP-43/PGP9.5 ratio $>95$ th percentile of the control individuals was $1 / 25(4.0 \%)$ in the Control 2 group and $9 / 34(26.5 \%)$ in the DSPN+p group $(p<0.05)$.

Inverse correlations between PGP9.5 positive IENFD and DNFL GAP-43/PGP9.5 ratio ( $\beta=-0.569, p<0.0001)$, as well as PGP9.5 positive DNFL and DNFL GAP-43/PGP9.5 ratio $(\beta=-0.639, p<0.0001)$, were found for all individuals with type 2 diabetes. In contrast, no such correlations were observed in the control individuals $(\beta=0.052, p=0.697$; $\beta=-0.127, p=0.344$, respectively).

The correlations of cutaneous nerve fibre density and length with measures of large and small fibre function are shown in Table 3. After adjustment for sex, age and BMI, GAP-43 positive IENFD was positively associated with sural sensory NCV and sural SNAPs in both DSPN groups, and also positively associated with peroneal motor NCV and cold TDT and inversely associated with warm TDT in the DSPN $+\mathrm{p}$ group $(p<0.05)$. GAP-43 positive DNFL was positively associated with peroneal motor NCV in the DSPN+p group, and with sural sensory NCV and sural SNAPs in both DSPN 
Table 2 IENFD, IENFL and DNFL immunostained with PGP9.5 and GAP-43

\begin{tabular}{|c|c|c|c|c|c|}
\hline & Control 1 & $\begin{array}{l}\text { Recent-onset type } \\
2 \text { diabetes }\end{array}$ & Control 2 & DSPN-p & $\mathrm{DSPN}+\mathrm{p}$ \\
\hline \multicolumn{6}{|l|}{ PGP9.5 } \\
\hline $\begin{array}{l}\text { IENFD } \\
(\text { fibres } / \mathrm{mm})\end{array}$ & $7.76 \pm 3.28$ & $5.11 \pm 2.96^{*}$ & $7.01 \pm 3.07$ & $3.81 \pm 3.85^{\dagger}$ & $2.43 \pm 2.86^{\dagger}$ \\
\hline IENFL $\left(\mu \mathrm{m} / \mathrm{mm}^{2}\right)$ & $6.21 \pm 3.57$ & $2.65 \pm 1.69^{*}$ & $5.09 \pm 2.73$ & $2.89 \pm 3.34^{\dagger}$ & $2.09 \pm 3.09^{\dagger}$ \\
\hline DNFL $\left(\mu \mathrm{m} / \mathrm{mm}^{2}\right)$ & $6.29 \pm 2.09$ & $5.84 \pm 1.59$ & $5.22 \pm 1.31$ & $4.72 \pm 2.28$ & $3.85 \pm 2.67$ \\
\hline \multicolumn{6}{|l|}{ GAP-43 } \\
\hline $\begin{array}{l}\text { IENFD } \\
(\text { fibres } / \mathrm{mm})\end{array}$ & $7.20 \pm 3.23$ & $4.86 \pm 2.67 *$ & $6.65 \pm 3.37$ & $3.48 \pm 3.28^{\dagger}$ & $2.29 \pm 2.61^{\dagger}$ \\
\hline IENFL $\left(\mu \mathrm{m} / \mathrm{mm}^{2}\right)$ & $4.93 \pm 3.29$ & $1.98 \pm 1.09 *$ & $4.06 \pm 2.71$ & $2.07 \pm 2.62^{\dagger}$ & $1.44 \pm 2.07^{\dagger}$ \\
\hline DNFL $\left(\mu \mathrm{m} / \mathrm{mm}^{2}\right)$ & $6.16 \pm 1.96$ & $5.60 \pm 1.46$ & $5.30 \pm 1.23$ & $5.00 \pm 2.19$ & $4.15 \pm 2.60$ \\
\hline \multicolumn{6}{|l|}{ GAP-43/PGP9.5 ratio } \\
\hline IENFD & $0.92 \pm 0.12$ & $1.00 \pm 0.23$ & $0.93 \pm 0.13$ & $0.96 \pm 0.15$ & $1.08 \pm 0.44$ \\
\hline IENFL & $0.80 \pm 0.30$ & $0.83 \pm 0.23$ & $0.82 \pm 0.35$ & $0.87 \pm 0.40$ & $1.25 \pm 1.99$ \\
\hline DNFL & $0.99 \pm 0.09$ & $0.97 \pm 0.11$ & $1.02 \pm 0.10$ & $1.07 \pm 0.10^{\dagger}$ & $1.18 \pm 0.28^{\ddagger}, 8$ \\
\hline
\end{tabular}

$* p<0.05$ vs Control $1,{ }^{\dagger} p<0.05$ vs Control $2,{ }^{\ddagger} p=0.05$ vs Control $2,{ }^{\S} p<0.05$ vs DSPN-p (all adjusted for sex, age, $\mathrm{BMI}$ and $\mathrm{HbA}_{1 \mathrm{c}}$ )

groups $(p<0.05)$. The correlations of PGP9.5 positive fibres with measures of large and small fibre function corresponded to those found for GAP-43 (data not shown). The DNFL GAP-43/PGP ratio was inversely associated with sural SNAPs in the DSPN-p group $(p<0.05)$. In the entire type 2 diabetes group, all variables of cutaneous nerve fibre density and length were associated with each measure of large and small fibre function $(p<0.05)$. No such associations were noted for the control participants (Table 3) or in the recentonset group (data not shown).
Table 3 Correlations of cutaneous nerve fibre density and length with measures of large and small fibre function

\begin{tabular}{|c|c|c|c|c|c|c|c|c|}
\hline & \multicolumn{2}{|l|}{ Control } & \multicolumn{2}{|l|}{ DSPN-p } & \multicolumn{2}{|c|}{$\mathrm{DSPN}+\mathrm{p}$} & \multicolumn{2}{|c|}{$\begin{array}{l}\text { All individuals } \\
\text { with diabetes }\end{array}$} \\
\hline & $r$ & $p$ & $r$ & $p$ & $r$ & $p$ & $r$ & $p$ \\
\hline \multicolumn{9}{|l|}{$\log _{e}($ IENFD-GAP-43) } \\
\hline $\begin{array}{l}\text { Peroneal } \\
\text { motor NCV }\end{array}$ & 0.263 & 0.071 & 0.324 & 0.099 & 0.579 & $0.001 *$ & 0.351 & $0.002 *$ \\
\hline $\begin{array}{l}\text { Sural sensory } \\
\text { NCV }\end{array}$ & 0.366 & 0.011 & 0.574 & $0.005^{*}$ & 0.554 & $0.005^{*}$ & 0.473 & $<0.0001 *$ \\
\hline $\begin{array}{l}\log _{e} \\
\quad \text { (sural SNAP) }\end{array}$ & 0.376 & 0.008 & 0.587 & $0.004 *$ & 0.455 & $0.025^{*}$ & 0.456 & $<0.0001^{*}$ \\
\hline $\log _{\mathrm{e}}(\mathrm{VPT})$ & -0.421 & 0.003 & 0.026 & 0.890 & -0.222 & 0.222 & -0.281 & $0.010 *$ \\
\hline $\begin{array}{l}\log _{e} \\
\quad \text { (warm TDT) }\end{array}$ & -0.194 & 0.187 & -0.129 & 0.481 & -0.523 & $0.001 *$ & -0.257 & $0.016^{*}$ \\
\hline $\begin{array}{l}\log _{\mathrm{e}} \\
\quad(\text { cold TDT })\end{array}$ & 0.330 & 0.025 & 0.094 & 0.610 & 0.621 & $<0.0001 *$ & 0.257 & $0.016^{*}$ \\
\hline \multicolumn{9}{|l|}{ DNFL GAP-43 } \\
\hline $\begin{array}{l}\text { Peroneal } \\
\text { motor NCV }\end{array}$ & 0.175 & 0.234 & 0.365 & 0.059 & 0.569 & $0.001 *$ & 0.489 & $<0.0001^{*}$ \\
\hline $\begin{array}{l}\text { Sural sensory } \\
\text { NCV }\end{array}$ & 0.181 & 0.224 & 0.579 & $0.005 *$ & 0.516 & $0.01 *$ & 0.517 & $<0.0001^{*}$ \\
\hline $\begin{array}{l}\log _{\mathrm{e}} \\
\quad \text { (sural SNAP) }\end{array}$ & 0.180 & 0.217 & 0.692 & $0.0004 *$ & 0.534 & $0.007 *$ & 0.588 & $<0.0001^{*}$ \\
\hline \multicolumn{9}{|l|}{ DNFL ratio } \\
\hline $\begin{array}{l}\log _{\mathrm{e}} \\
\quad \text { (sural SNAP) }\end{array}$ & -0.163 & 0.262 & -0.548 & $0.008 *$ & -0.037 & 0.862 & -0.359 & $0.001 *$ \\
\hline $\begin{array}{l}\log _{\mathrm{e}} \\
\quad(\text { cold TDT) }\end{array}$ & -0.100 & 0.507 & -0.199 & 0.276 & -0.446 & 0.008 & -0.433 & $<0.0001^{*}$ \\
\hline
\end{tabular}

$* p<0.05$ after adjustment for sex, age and BMI 


\section{Discussion}

In this comprehensive study using PGP9.5 and GAP-43 double staining, we demonstrate differential patterns of cutaneous nerve fibre loss and regeneration in various groups of individuals with type 2 diabetes with and without painful DSPN. First, dermal nerve fibre regeneration assessed by the DNFL GAP-43/PGP9.5 ratio was enhanced in both types of DSPN, to a higher extent in individuals with DSPN $+p$ compared with those with DSPN-p. Second, the degree of dermal nerve fibre regeneration was associated with more advanced intraepidermal nerve fibre loss and peripheral nerve dysfunction. Third, the IENFDGAP-43/PGP9.5 ratio did not differ between the groups, questioning the usefulness of this ratio as a marker of nerve regeneration in type 2 diabetes. Thus, we suggest that, despite progressive epidermal nerve fibre loss, dermal nerve repair remains stimulated in DSPN, particularly in DSPN $+p$, but fails to adequately counteract epidermal neurodegenerative processes.

A major finding presented herein is the increased DNFL GAP-43/PGP9.5 ratio in DSPN, suggesting an increased attempt at regeneration by dermal nerve fibres, particularly in DSPN+p. Although apparently contradictory to the common assumption that regenerative capacity is reduced in individuals with diabetes $[4,19]$, these results are in line with basic principles of neural plasticity in the peripheral nervous system. Physiologically, nerve remodelling and regrowth are part of an intact peripheral neural plasticity [14], representing processes in which numerous extrinsic and intrinsic factors, such as GAP-43, are involved. These factors are fairly robust against degenerative events in cutaneous sensory nerve fibres [29]. Following nerve injury, GAP-43 is strongly upregulated in the axonal growth cone at the distal end of the proximal axonal stump [4], to compensate for axonal loss. While GAP43 may not exclusively label regenerating nerve fibres, its preponderance in the regenerating growth cones compared with degenerating distal axonal stumps or uninjured axons justifies its use as a post-injury regenerative marker [4]. Therefore, the increase in GAP-43 DNFL relative to PGP9.5 DNFL in individuals with DSPN and the inverse association with IENFD could mirror a compensatory upregulation against the neurodegenerative effects of diabetes which ultimately may be insufficient to increase IENFD in a diabetesinduced microenvironment inhospitable for axonal regrowth [30]. Interestingly, an enhanced DNFL GAP-43/PGP9.5 ratio was associated with more advanced intraepidermal nerve fibre loss and peripheral nerve dysfunction in individuals with type 2 diabetes, whereas no such association was found in control participants. Together, these skin biopsy measures correlated with large fibre function (NCV) for both types of DSPN, while they were only associated with small fibre function (TDT) in $\mathrm{DSPN}+\mathrm{p}$. These findings support the concept of a higher rate of maladaptive small fibre regeneration processes in DSPN $+p$ [31-33], which may result in enhanced pain perception due to peripheral sensitisation. Supporting this rationale, a recent study by Xie et al [34] reported that nerve regeneration in a spinal nerve injury model resulted in tangled GAP-43 positive neuromas and that blocking these regenerative processes reduced neuropathic pain.

To the best of our knowledge, this is the first study in which both IENFL and DNFL were quantified manually in adequately large groups of individuals with type 2 diabetes and control participants using double immunofluorescence for PGP9.5 and GAP-43. Direct comparisons of the various aspects of this study with previous reports are difficult because of differences in methodology and aetiology of neuropathy. For example, previous studies have not specified the proportion of individuals with DSPN $[18,28,35,36]$, have used bright-field microscopy $[8,37,38]$ or relied on automatic measurement of nerve fibre density [16]. Focusing on IENFD, Cheng et al [17] found no difference in IENFD for PGP9.5 positive fibres between DSPN+p and DSPN-p but, in contrast to our findings, they reported more GAP-43 positive fibres in DSPN $+p$ and reduced intraepidermal GAP-43/ PGP9.5 ratios, particularly in DSPN-p, compared with individuals without DSPN. A previous study by Sorensen et al [39] showed reduced PGP9.5 positive IENFD in people with DSPN+p compared with a group of individuals without pain, but it is unclear how many individuals in the latter group had DSPN. Compatible with our data, Themistocleous et al [38] reported similarly reduced PGP9.5 positive IENFD in both DSPN entities using bright-field microscopy. However, $30 \%$ of the DSPN-p group were receiving analgesic pharmacotherapy [38], casting doubt on the validity of the DSPN-p group.

In contrast to previous studies, we found no evidence of dermal nerve fibre loss in the three type 2 diabetes groups studied. In a small study, Krishnan et al [37] reported reduced dermal nerve fibre density in DSPN-p but not DSPN+p using only $5 \mu \mathrm{m}$ thin sections. Lauria et al [28] found reduced DNFL in individuals with SFN, but they neither reported the proportions of participants with diabetic vs idiopathic SFN nor did they discriminate between painful and painless SFN.

The higher proportion of GAP-43 compared with PGP9.5 positive cutaneous nerve fibres found in the DSPN groups is challenging given the recognised role of PGP9.5 as a pan-neuronal marker. A possible explanation could be that GAP-43 is superior to PGP9.5 in detecting emerging nerve fibre endings because of different axonal transport mechanisms. PGP9.5 is an enzyme that relies on the slow axonal transport component B (2-8 mm/day), while GAP-43 uses fast vesicular axonal transport (50$400 \mathrm{~mm} /$ day) $[12,40]$. Hence, GAP-43 could be more susceptible than PGP9.5 to recently grown nerve fibres. This could be an advantage in areas of high neural plasticity and needs to be evaluated in further studies. 
Major strengths of this work are the manual assessment of both epidermal and dermal nerve fibres using double immunofluorescence microscopy including sophisticated morphometry and the extensive functional phenotyping performed in relatively large samples of individuals with type 2 diabetes and control participants. One limitation is the crosssectional nature of this study which does not give information on the temporal sequence of the findings presented. Another limitation is the restriction to largely descriptive morphological and functional assessment which may not provide direct insights into the pathological mechanisms. Moreover, topical capsaicin application and excision axotomy models have been introduced to study nerve regeneration $[19,30]$, but these require sampling of multiple serial skin biopsies usually feasible only in a limited number of individuals.

In conclusion, the regenerative capacity of dermal nerve fibres assessed by the DNFL GAP-43/PGP9.5 ratio was enhanced in both types of DSPN, particularly in DSPN+p, but not in individuals with recent-onset type 2 diabetes. The extent of the dermal regenerative capacity was associated with more advanced intraepidermal nerve fibre loss and peripheral nerve dysfunction. We propose that the DNFL GAP-43/PGP9.5 ratio should be considered as a marker to assess the regenerative capacity of cutaneous nerve fibres. Whether this biomarker has the potential to predict the progression or regression of DSPN or the susceptibility to regenerative pharmacotherapy remains to be established in future prospective observational studies and clinical trials.

Acknowledgements The authors wish to thank the staff of the Research Group Neuropathy, Institute for Clinical Diabetology at the German Diabetes Center (DDZ), Düsseldorf, Germany, especially F. Battiato, N. Reuß and M. Schroers-Teuber, for their excellent work. The GDS Group consists of A.E. Buyken (Department of Sports and Health, Paderborn University, Paderborn, Germany), G. Geerling (Department of Ophthalmology, Medical Faculty, Heinrich Heine University, Düsseldorf, Germany), J. Eckel, H. Al-Hasani, C. Herder, A. Icks, J. Kotzka, O. Kuss, E. Lammert, D. Markgraf, K. Müssig, W. Rathmann, J. Szendroedi, D. Ziegler and M. Roden (speaker) (all DDZ). Some of the data were presented as an abstract at the 52nd EASD Annual Meeting in Munich in 2016.

Data availability The data sets generated during and/or analysed during the current study are not publicly available, since they are subject to national data protection laws and restrictions imposed by the ethics committee to ensure data privacy of the study participants. However, they can be applied for through an individual project agreement with PROPANE and/or GDS.

Funding The GDS was initiated and financed by the German Diabetes Center, which is funded by the German Federal Ministry of Health (Berlin, Germany), the Ministry of Innovation, Science, Research and Technology of the state North Rhine-Westphalia (Düsseldorf, Germany), grants from the German Federal Ministry of Education and Research (BMBF) to the German Center for Diabetes Research (DZD), and partly funded through an EFSD award supported by Novartis to DZ and AS. The PROPANE study was initiated by the PROPANE consortium and received funding from the European Union Seventh Framework Programme FP7/2007-2013 (grant no. 602273).

Duality of interest The authors declare that there is no duality of interest associated with this manuscript.

Contribution statement All authors were involved in revising the manuscript critically for important intellectual content and gave final approval of the version to be published. GJB contributed to acquisition, analysis and interpretation of data and wrote the manuscript. AS and SP contributed to the acquisition, analysis and interpretation of data, BR and $\mathrm{JB}$ contributed to the acquisition of data. KB, KM, JS and MR contributed to the analysis and interpretation of data. DZ contributed to conception and design of the study and to the analysis and interpretation of data. DZ is the guarantor of this work and, as such, had full access to all the data in the study and takes responsibility for the integrity of the data and the accuracy of the data analysis.

\section{References}

1. Happich M, John J, Stamenitis S, Clouth J, Polnau D (2008) The quality of life and economic burden of neuropathy in diabetic patients in Germany in 2002-results from the Diabetic Microvascular Complications (DIMICO) study. Diabetes Res Clin Pract 81:223-230

2. Ziegler D, Papanas N, Vinik AI, Shaw JE (2014) Epidemiology of polyneuropathy in diabetes and prediabetes. Handb Clin Neurol 126:3-22

3. Landowski LM, Dyck PJ, Engelstad J, Taylor BV (2016) Axonopathy in peripheral neuropathies: mechanisms and therapeutic approaches for regeneration. J Chem Neuroanat 76:19-27

4. Yasuda H, Terada M, Maeda K et al (2003) Diabetic neuropathy and nerve regeneration. Prog Neurobiol 69:229-285

5. Zochodne DW (2012) The challenges and beauty of peripheral nerve regrowth. J Peripher Nerv Syst 17:1-18

6. Spallone V, Greco C (2013) Painful and painless diabetic neuropathy: one disease or two? Curr Diab Rep 13:533-549

7. Tesfaye S, Vileikyte L, Rayman G et al (2011) Painful diabetic peripheral neuropathy: consensus recommendations on diagnosis, assessment and management. Diabetes Metab Res Rev 27:629-638

8. Lauria G, Lombardi R, Camozzi F, Devigili G (2009) Skin biopsy for the diagnosis of peripheral neuropathy. Histopathology 54:273285

9. Day IN, Thompson RJ (2010) UCHL1 (PGP 9.5): neuronal biomarker and ubiquitin system protein. Prog Neurobiol 90:327-362

10. Ziegler D, Papanas N, Zhivov A et al (2014) Early detection of nerve fiber loss by corneal confocal microscopy and skin biopsy in recently diagnosed type 2 diabetes. Diabetes 63:2454-2463

11. Kennedy WR, Wendelschafer-Crabb G, Johnson T (1996) Quantitation of epidermal nerves in diabetic neuropathy. Neurology 47:1042-1048

12. Denny JB (2006) Molecular mechanisms, biological actions, and neuropharmacology of the growth-associated protein GAP-43. Curr Neuropharmacol 4:293-304

13. Benowitz LI, Routtenberg A (1997) GAP-43: an intrinsic determinant of neuronal development and plasticity. Trends Neurosci 20 : 84-91

14. Verze L, Viglietti-Panzica C, Maurizo S, Sica M, Panzica G (2003) Distribution of GAP-43 nerve fibers in the skin of the adult human hand. Anat Rec A Discov Mol Cell Evol Biol 272:467-473

15. Fantini F, Johansson O (1992) Expression of growth-associated protein 43 and nerve growth factor receptor in human skin: a 
comparative immunohistochemical investigation. J Invest Dermatol 99:734-742

16. Narayanaswamy H, Facer P, Misra VP et al (2012) A longitudinal study of sensory biomarkers of progression in patients with diabetic peripheral neuropathy using skin biopsies. J Clin Neurosci 19: 1490-1496

17. Cheng HT, Dauch JR, Porzio MT et al (2013) Increased axonal regeneration and swellings in intraepidermal nerve fibers characterize painful phenotypes of diabetic neuropathy. J Pain 14:941-947

18. Bursova S, Dubovy P, Vlckova-Moravcova E et al (2012) Expression of growth-associated protein 43 in the skin nerve fibers of patients with type 2 diabetes mellitus. J Neurol Sci 315:60-63

19. Polydefkis M, Hauer P, Sheth S, Sirdofsky M, Griffin JW, McArthur JC (2004) The time course of epidermal nerve fibre regeneration: studies in normal controls and in people with diabetes, with and without neuropathy. Brain 127:1606-1615

20. ADA (2012) Diagnosis and classification of diabetes mellitus. Diabetes Care 35(Suppl 1):S64-S71

21. Tesfaye S, Boulton AJ, Dyck PJ et al (2010) Diabetic neuropathies: update on definitions, diagnostic criteria, estimation of severity, and treatments. Diabetes Care 33:2285-2293

22. Dworkin RH, Turk DC, Peirce-Sandner S et al (2010) Research design considerations for confirmatory chronic pain clinical trials: IMMPACT recommendations. Pain 149:177-193

23. Szendroedi J, Saxena A, Weber KS et al (2016) Cohort profile: the German Diabetes Study (GDS). Cardiovasc Diabetol 15:59

24. Lauria G, Ziegler D, Malik R et al (2014) The role of sodium channels in painful diabetic and idiopathic neuropathy. Curr Diab Rep 14:538

25. Young MJ, Boulton AJ, MacLeod AF, Williams DR, Sonksen PH (1993) A multicentre study of the prevalence of diabetic peripheral neuropathy in the United Kingdom hospital clinic population. Diabetologia 36:150-154

26. Lauria G, Hsieh ST, Johansson O et al (2010) European Federation of Neurological Societies/Peripheral Nerve Society guideline on the use of skin biopsy in the diagnosis of small fiber neuropathy. Report of a joint task force of the European Federation of Neurological Societies and the Peripheral Nerve Society. Eur J Neurol 17(903912):e944-e909

27. McCarthy BG, Hsieh ST, Stocks A et al (1995) Cutaneous innervation in sensory neuropathies: evaluation by skin biopsy. Neurology 45:1848-1855
28. Lauria G, Cazzato D, Porretta-Serapiglia C et al (2011) Morphometry of dermal nerve fibers in human skin. Neurology 77:242-249

29. Cheng C, Guo GF, Martinez JA, Singh V, Zochodne DW (2010) Dynamic plasticity of axons within a cutaneous milieu. J Neurosci 30:14735-14744

30. Ebenezer GJ, O'Donnell R, Hauer P, Cimino NP, McArthur JC, Polydefkis M (2011) Impaired neurovascular repair in subjects with diabetes following experimental intracutaneous axotomy. Brain 134:1853-1863

31. Costigan M, Scholz J, Woolf CJ (2009) Neuropathic pain: a maladaptive response of the nervous system to damage. Annu Rev Neurosci 32:1-32

32. Orstavik K, Namer B, Schmidt R et al (2006) Abnormal function of C-fibers in patients with diabetic neuropathy. J Neurosci 26:1128711294

33. Navarro X, Vivo M, Valero-Cabre A (2007) Neural plasticity after peripheral nerve injury and regeneration. Prog Neurobiol 82:163201

34. Xie W, Strong JA, Zhang JM (2017) Active nerve regeneration with failed target reinnervation drives persistent neuropathic pain. eNeuro. https://doi.org/10.1523/ENEURO.0008-17.2017

35. Scheytt S, Riediger N, Braunsdorf S, Sommer C, Uceyler N (2015) Increased gene expression of growth associated protein- 43 in skin of patients with early-stage peripheral neuropathies. J Neurol Sci 355:131-137

36. Vlckova-Moravcova E, Bednarik J, Dusek L, Toyka KV, Sommer C (2008) Diagnostic validity of epidermal nerve fiber densities in painful sensory neuropathies. Muscle Nerve 37:50-60

37. Krishnan ST, Quattrini C, Jeziorska M, Malik RA, Rayman G (2009) Abnormal LDIflare but normal quantitative sensory testing and dermal nerve fiber density in patients with painful diabetic neuropathy. Diabetes Care 32:451-455

38. Themistocleous AC, Ramirez JD, Shillo PR et al (2016) The Pain in Neuropathy Study (PiNS): a cross-sectional observational study determining the somatosensory phenotype of painful and painless diabetic neuropathy. Pain 157:1132-1145

39. Sorensen L, Molyneaux L, Yue DK (2006) The relationship among pain, sensory loss, and small nerve fibers in diabetes. Diabetes Care 29:883-887

40. Rage M, Van Acker N, Facer P et al (2010) The time course of $\mathrm{CO}_{2}$ laser-evoked responses and of skin nerve fibre markers after topical capsaicin in human volunteers. Clin Neurophysiol 121:1256-1266 